Research Article

\title{
Clinical Features and Rules of Chinese Herbal Medicine in Diabetic Peripheral Neuropathy Patients
}

\author{
Jindong Zhao $\mathbb{D}^{1},{ }^{1}$ Yan Li, ${ }^{2}$ Ling Xin, ${ }^{3}$ Min Sun $\mathbb{D},{ }^{4}$ Chanjuan Yu, ${ }^{1}$ Guobin Shi, ${ }^{1}$ Taotao Bao, \\ Jian Liu, ${ }^{1}$ Yingqun Ni, ${ }^{1}$ RuiMin Lu, ${ }^{1}$ Yuanyuan $\mathrm{Wu}^{1}{ }^{1}$ and Zhaohui Fang $\mathbb{D}^{1}$ \\ ${ }^{1}$ Department of Endocrine Disease, The First Affiliated Hospital of Anhui University of Chinese Medicine, Hefei 230031, China \\ ${ }^{2}$ Department of Infectious Disease, The First Affiliated Hospital of Anhui University of Chinese Medicine, Hefei 230031, China \\ ${ }^{3}$ Department of Information, The First Affiliated Hospital of Anhui University of Chinese Medicine, Hefei 230031, China \\ ${ }^{4}$ School of Life Sciences, Anhui University, Hefei 230039, China
}

Correspondence should be addressed to Zhaohui Fang; fangzhaohui1111@163.com

Received 10 December 2019; Revised 27 March 2020; Accepted 8 June 2020; Published 17 July 2020

Academic Editor: Mark Moss

Copyright (c) 2020 Jindong Zhao et al. This is an open access article distributed under the Creative Commons Attribution License, which permits unrestricted use, distribution, and reproduction in any medium, provided the original work is properly cited.

\begin{abstract}
Objective. To analyse the clinical features of diabetic peripheral neuropathy (DPN) and employ data mining technology to explore the rules of Chinese herbal medicine (CHM) therapy. Methods. The clinical data of 216 patients with DPN and qi-yin deficiency syndrome were obtained, and the clinical features of the patients were assessed by cluster analysis. Relevant information was entered into the clinical diagnosis and treatment collection system, and data mining techniques were used to analyse the drug frequency, core CHM, CHM pair, and so on. Results. In this study, glycated haemoglobin (HbAlc) and homocysteine (HCY) were closely related to the pathogenesis of DPN. Overall, 162 patients had typical DPN syndrome characteristics, and we analysed 216 prescriptions, including $182 \mathrm{CHM}$. The frequencies of prescription of Astragalus membranaceus, Ligusticum wallichii, Poria cocos, and Radix Rehmanniae were greater than $45 \%$. A Bayesian network analysis diagram showed that the 9 most common core CHM included Astragalus membranaceus, Ligusticum wallichii, Poria cocos, atractylodes rhizome, and Salvia miltiorrhiza Bge. According to the association rules of CHM, Radix Ophiopogon is used for Codonopsis pilosula; Astragalus membranaceus and atractylodes rhizome for Rehmannia are also frequently used. Astragalus membranaceus and Cinnamomi Ramulus or Ligusticum wallichii and Moutan bark were highly related to a decreased Michigan Diabetic Neuropathy Score. Conclusion. HbA1c and HCY are related risk factors for DPN. Numbness is a typical syndrome characteristic. Astragalus membranaceus is a monarch CHM and is used most frequently.
\end{abstract}

\section{Introduction}

The incidence of diabetic peripheral neuropathy (DPN) is approximately $10-96 \%$ and is one of the more common chronic complications of diabetes [1]. The main causes of DPN are metabolic disorders and vascular damage [2]. DPN presents as a wide spectrum of symptoms, such as sensory loss, pain, and loss of muscle mass [3]. Although nerve conduction velocity is common in DPN patients, it is not easily or reliably diagnosed in DPN [4]. However, $10 \mathrm{~g}$ of nylon yarn, a $128 \mathrm{~Hz}$ tuning fork, the Leeds Assessment of Neuropathic Symptoms and Signs, the Toronto Clinical Scoring System, and other tools have been widely used in the diagnosis of DPN [5-8]. Currently, the
Michigan Diabetic Neuropathy Score (MDNS) is also a commonly used tool for diagnosing DPN [9].

Some research has proven the efficacy of Chinese herbal medicine (CHM) in the treatment of DPN [10-12]. Thus, we believe that Traditional Chinese Medicine (TCM) has potential in the prevention and treatment of DPN. The pathogenesis of DPN is qi-yin deficiency in TCM, and this pattern gradually develops into a yin and yang deficiency. Blood stasis is a sign in the blood vessels and results in pain and itching [13]. We have recorded the general information of DPN patients and the prescription of CHM treatment based on real-world applications. This study provides a summary of patients' clinical characteristics and experience with CHM. 


\section{Subjects and Methods}

2.1. Patient Recruitment. The patients selected for this study included 216 patients with DPN with qi-yin deficiency syndrome who were treated with CHM, which included 216 prescriptions, from January 2016 to December 2019 at the First Affiliated Hospital of Anhui University of Chinese Medicine. Patients for whom treatment was effective were included. The main basis for evaluating clinical efficacy was the improvement in the subjective symptoms of patients, such as numbness, coldness and pain, and a decreased MDNS [14].

2.2. DPN Diagnostic Criteria. This study referred to the DPN diagnostic criteria [1] as follows: history of diabetes; numbness, pain, chills, burning, or other abnormal feelings as the main symptoms; an electromyogram indicating that the nerve conduction speed had slowed down or that the latency period was prolonged; and a MDNS indicating nerve damage.

2.3. Diagnostic Criteria for Qi-Yin Deficiency Syndrome. According to the "Guiding Principles for Clinical Research of New Chinese Medicine in the Treatment of Diabetes" [14] and the Diabetes Branch of the Chinese Medicine Association "Guidelines for TCM Clinical Diagnosis and Treatment of DPN" [15], the criteria for qi-yin deficiency syndrome are as follows: numbness; pain (cold or burning); electric shock; tingling; pain at night; pins and needles feeling; tiredness; dry skin; a sense of decreased pain; a dark, purple, or spotted tongue; and a fine or astringent pulse.

2.4. Inclusion Criteria. The inclusion criteria were as follows: patients who met the DPN diagnostic criteria with qi-yin deficiency syndrome and patients whose medical records, prescription medication data, and other information were complete.

2.5. Exclusion Criteria. The following patients were excluded: patients with peripheral neuropathy caused by other diseases; patients who were unwilling to cooperate (could not cooperate with dietary control or did not use medicines as prescribed); patients with severe acute or chronic complications of diabetes or other serious primary diseases; patients who did not take CHM regularly; or patients for whom clinical efficacy could not be evaluated.

2.6. Treatment. Patients followed the diabetes diet, exercised regularly, and took CHM in the morning and evening based on the conventional treatment for DPN.

2.7. Establishment of the Database and Regulating Data. Information on age, sex, body mass index (BMI), MDNS, and biochemical indexes of DPN patients with qi-yin deficiency syndrome was entered into an Excel database. The CHM database was established using the "Diabetes Clinical Medical Case Diagnosis and Treatment Information Collection System" developed by The First Affiliated Hospital of
Anhui University of Chinese Medicine. This database was base on Xuezhong Zhou's platform [16]. This platform promotes the development of TCM from individualized empirical knowledge to evidence-based medicine.

Medical records were entered by trained physicians and then checked by the quality control staff of the research team to ensure the accuracy of the information. The names of CHM prescriptions were standardized according to the 2015 edition of the "Pharmacopoeia of the People's Republic of China" [17].

2.8. Statistical Analyses. Statistical analyses were performed using SPSS 23.0 statistical software (SPSS Inc., Chicago, IL, USA). Measurement data with a normal distribution are expressed as the mean \pm standard deviation (SD), and the squared deviation method was used for cluster analysis. The CHM rules were analysed with the a priori algorithm, entropy clustering of complex systems, and Bayesian network analysis. The prediction accuracy of the machine learning classification methods including association rules, cluster analysis, and complex networks using clinical data were $84.1 \%, 75.0 \%$, and $84.5 \%$, respectively $[16,18] . P<0.05$ was considered to indicate statistical significance.

\section{Results}

3.1. Comparison of Baseline Characteristics. Of the 216 patients, 152 patients were male, and 64 patients were female. The baseline characteristics of patients are shown in Table 1. The data were divided into two groups by duration of diabetes (DOD) of 10 years or more, and the characteristics of two groups are shown in Table 2. Cluster analysis was performed on the baseline characteristics. When the abscissa was set to 10 , data for low-density lipoprotein cholesterol (LDL-C), MDNS, triglycerides (TG), total cholesterol (TC), fasting plasma glucose (FPG), glycated haemoglobin (HbAlc), age, DOD, BMI, and homocysteine (HCY) were obtained. The results are shown in Figure 1.

3.2. Typical Syndrome Characteristics. There were 162 patients who had typical syndrome characteristics of DPN and 54 patients who had no symptoms. The frequencies of the typical syndrome characteristics are shown in Figure 2. Among the characteristics, numbness occurred most often.

3.3. CHM Frequency. A total of 182 types of CHM were used, and the top 20 types are listed in Table 3. Astragalus membranaceus was the most frequently used, with a frequency of $67.12 \%$ in 216 prescriptions. Among the CHM, more than $45 \%$ were Ligusticum wallichii, Poria cocos, and Radix Rehmanniae. The highest dosage of Polygonatum kingianum Coll. et Hemsl was $28 \mathrm{~g}$.

3.4. CHM Characteristics. There were 64 warm herbals, 59 cold, 34 neutral, 10 cool, and 5 hot.

The following flavours were included: 94 were sweet, 80 were bitter, 59 were spicy, 17 were sour, 9 were light, 8 were salty, and 6 were astringent. 
Table 1: Baseline characteristics.

\begin{tabular}{lc}
\hline Variable & Value $($ mean \pm SD) \\
\hline Age (year) & $62.74 \pm 11.25$ \\
DOD (month) & $84.19 \pm 62.20$ \\
BMI $\left(\mathrm{kg} / \mathrm{m}^{2}\right)$ & $25.16 \pm 4.78$ \\
FPG $\left(\mathrm{mmol} \cdot \mathrm{L}^{-1}\right)$ & $8.54 \pm 4.01$ \\
HbAlc $(\%)$ & $9.91 \pm 2.86$ \\
TG $(\mathrm{mmol} / \mathrm{L})$ & $1.94 \pm 1.76$ \\
TC $(\mathrm{mmol} / \mathrm{L})$ & $4.59 \pm 1.03$ \\
LDL-C $(\mathrm{mmol} / \mathrm{L})$ & $2.59 \pm 1.03$ \\
HCY $(\mathrm{cm})$ & $18.98 \pm 22.40$ \\
MDNS (fraction) & $25.84 \pm 1.12$ \\
\hline
\end{tabular}

TABLE 2: Comparison of baseline characteristics in the two groups by DOD.

\begin{tabular}{lcccc}
\hline Variable & $\geq 10$ years & $<10$ years & Value & $P$ value \\
\hline Male/female & $42 / 21$ & $110 / 43$ & 0.585 & 0.512 \\
Age $($ year $)$ & $64.73 \pm 11.77$ & $61.92 \pm 10.97$ & 1.674 & 0.096 \\
BMI $\left(\mathrm{kg} / \mathrm{m}^{2}\right)$ & $24.70 \pm 4.36$ & $25.35 \pm 4.95$ & -0.916 & 0.361 \\
FPG $\left(\mathrm{mmol} \cdot \mathrm{L}^{-1}\right)$ & $8.00 \pm 3.91$ & $8.75 \pm 4.04$ & -1.253 & 0.212 \\
HbA1c $(\%)$ & $9.26 \pm 2.63$ & $10.17 \pm 2.91$ & -2.140 & $0.033 *$ \\
TG $(\mathrm{mmol} / \mathrm{L})$ & $1.75 \pm 1.42$ & $2.02 \pm 1.87$ & -1.015 & 0.311 \\
TC $(\mathrm{mmol} / \mathrm{L})$ & $4.44 \pm 0.94$ & $4.64 \pm 1.06$ & -1.342 & 0.181 \\
LDL-C $(\mathrm{mmol} / \mathrm{L})$ & $2.46 \pm 1.07$ & $2.63 \pm 1.00$ & -1.094 & 0.275 \\
HCY $(\mathrm{cm})$ & $17.79 \pm 18.99$ & $19.47 \pm 23.71$ & -0.051 & 0.617 \\
MDNS (fraction) & $25.73 \pm 5.25$ & $25.89 \pm 6.06$ & -0.061 & 0.951 \\
\hline
\end{tabular}

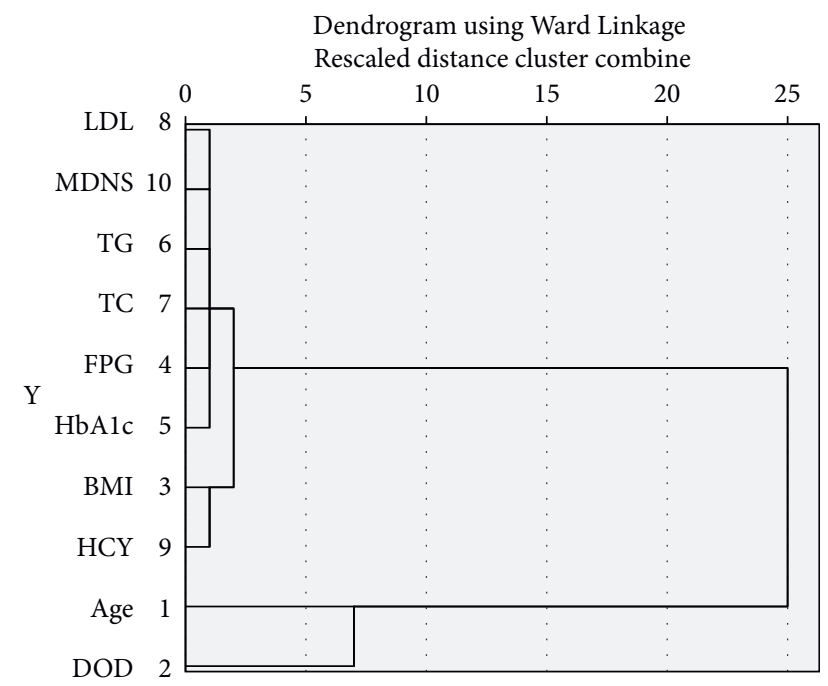

Figure 1: Cluster analysis from baseline characteristics.

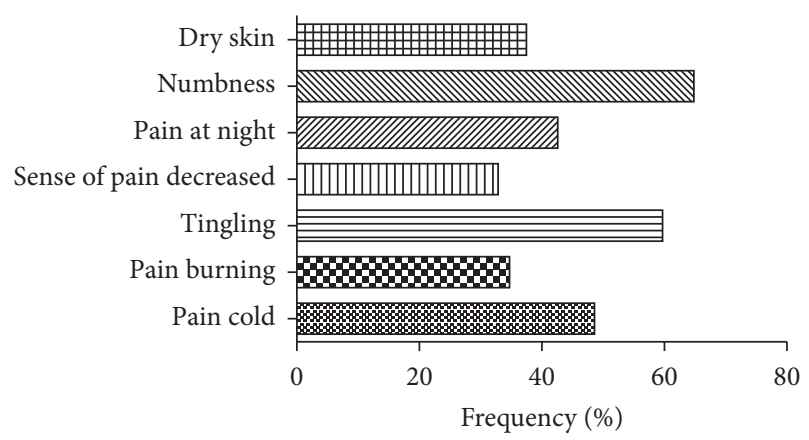

FIgURE 2: Typical syndrome characteristics.
There were 80 herbals for liver meridians, 72 for lung meridians, 67 for spleen meridians, 62 for kidney meridians, 40 for heart meridians, 18 for large bowel meridians, 14 for bladder meridians, 10 for small intestine meridians, 5 for pericardium meridians, and 3 for triple energizer meridians.

3.5. Core Prescriptions. Cluster analysis was performed on all CHM. The 9 most frequently CHM were Astragalus membranaceus, Ligusticum wallichii, Poria cocos, atractylodes rhizome, Salvia miltiorrhiza Bge., Angelica sinensis, Cinnamomi Ramulus, Carthamus tinctorius L., and Clematidis Radix et rhizome. The results are shown in Figure 3.

3.6. CHM Correlation. Correlation analysis was performed on all CHM. The support was $>80 \%$, and the confidence was $>90 \%$. There were 5 groups of CHM pairs, and there were 3 groups of CHM triads with support $\geq 75 \%$ and confidence $\geq 95 \%$. The results are shown in Table 4 .

3.7. CHM Cluster. Cluster analysis was performed on 20 types of CHM in terms of frequency. When the abscissa was set to 5, data on Typhonii Rhizoma-Asari Radix et RhizomaCinnamomi Ramulus-Clematidis Radix et Rhizoma-Carthamus tinctorius L., Ligusticum wallichii-Astragalus membranaceus, Poria cocos-atractylodes rhizome-Tangerine peel, Radix Rehmanniae-Radix Puerariae, Polygonatum kingianum Coll. et Hemsl-Achyranthes bidentata Bl.-Scutellaria baicalensis Georgi, and Paeoniae Radix Alba-Radix Ophiopogonis-Moutan bark were obtained. The results are shown in Figure 4.

3.8. CHM and Correlation Indicators. The analysis showed that CHM can lead to recognized evidence of improved microindicators by the application of association rules to mine correlations between CHM herbs and indicators. The results are shown in Table 5.

\section{Discussion}

HbAlc is an important indicator of glycaemic control among patients with diabetes. For most adult patients with diabetes, a reasonable target for $\mathrm{HbAlc}$ is $<7.0 \%$. Studies have shown that when HbAlc levels are $>8.0 \%$, the prevalence of DPN increases significantly [19]. HbA1c levels in this study were approximately $9.91 \%$, indicating that the glycaemic control of the patients was not good. Therefore, a high level of $\mathrm{HbA1c}$ is one of the main reasons that patients develop DPN with qi-yin deficiency syndrome. According to the comparison based on a DOD that had reached 10 years, patients with a long duration of disease had decreased $\mathrm{HbA1c}$ levels, likely due to the strict control of glycaemic as a result of fear of diabetic complications.

In this study, cluster analysis was performed on the baseline characteristics of patients to obtain 3 groups, including patients with average age and DOD. Some studies have shown that patients with a DOD of more than 10 years often have DPN [20]. However, the average DOD in this 
TABLe 3: CHM frequencies and dose.

\begin{tabular}{|c|c|c|c|c|c|}
\hline Drug name & Frequency (\%) & Dose $(\mathrm{g})$ & Drug name & Frequency (\%) & Dose $(\mathrm{g})$ \\
\hline Astragalus membranaceus & $145(67.12)$ & 24 & Tangerine peel & $74(34.26)$ & 12 \\
\hline Ligusticum wallichii & $109(50.46)$ & 15 & Radix Puerariae & $64(29.63)$ & 16 \\
\hline Poria cocos & $106(49.07)$ & 17 & Asari Radix et Rhizoma & $61(28.24)$ & 6 \\
\hline Radix Rehmanniae & $99(45.83)$ & 16 & Radix Ophiopogonis & $54(25.00)$ & 23 \\
\hline Atractylodes rhizome & $97(44.90)$ & 19 & Paeoniae Radix Alba & $53(24.54)$ & 25 \\
\hline Salvia miltiorrhiza Bge. & $89(41.20)$ & 10 & Moutan bark & $52(24.07)$ & 22 \\
\hline Angelica sinensis & $88(40.74)$ & 10 & Scutellaria baicalensis Georgi & $50(23.15)$ & 13 \\
\hline Cinnamomi Ramulus & $86(39.81)$ & 22 & Typhonii Rhizoma & $49(22.68)$ & 6 \\
\hline Carthamus tinctorius L. & $78(36.11)$ & 14 & Achyranthes bidentata $\mathrm{Bl}$ & $39(18.05)$ & 26 \\
\hline Clematidis Radix et Rhizoma & $77(35.64)$ & 27 & Polygonatum kingianum Coll. et Hemsl & $39(18.05)$ & 28 \\
\hline
\end{tabular}

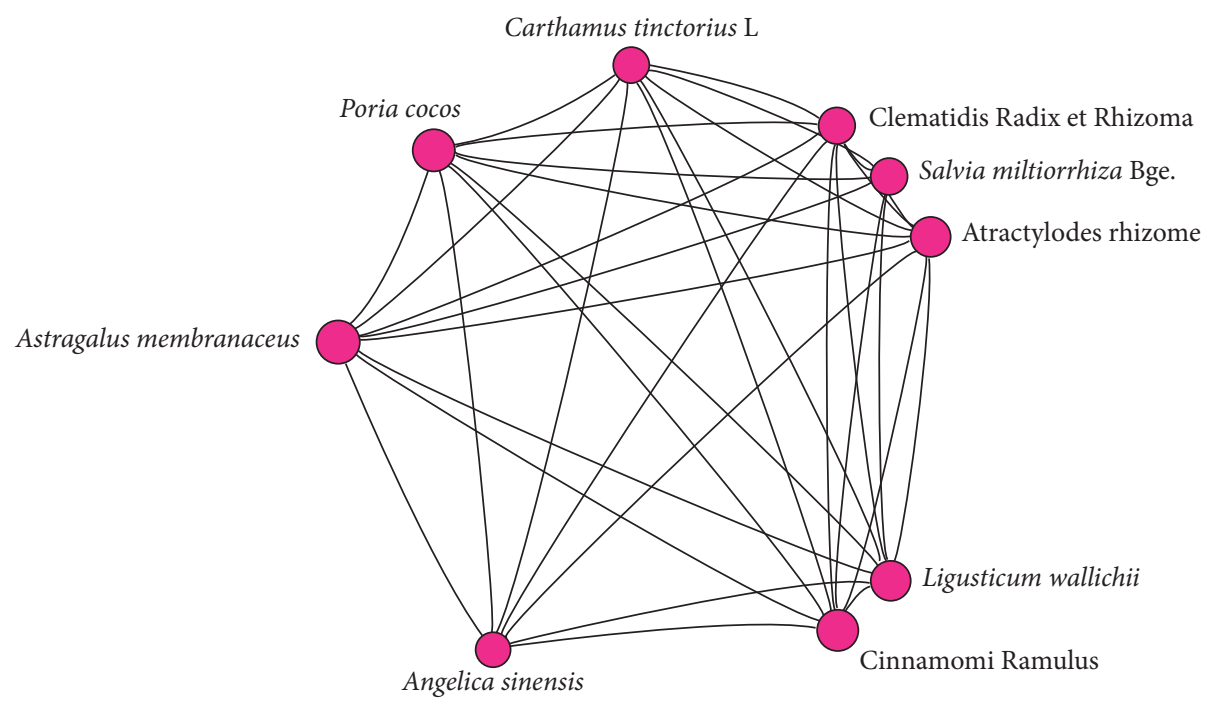

FIgURE 3: Core prescriptions.

TABLE 4: CHM correlation.

\begin{tabular}{lccc}
\hline & CHM pair & Support (\%) & Confidence (\%) \\
After item & Before item & 81.82 & 96.38 \\
Codonopsis pilosula & Radix Ophiopogonis & 80.63 & 95.84 \\
Scutellaria baicalensis Georgi & Radix Rehmanniae & 81.87 & 93.88 \\
Poria cocos & Atractylodes rhizome & 84.16 & 92.64 \\
Ligusticum wallichii & Angelica sinensis & 84.25 & 90.51 \\
Tangerine peel & Paeoniae Radix Alba & 76.86 & 98.65 \\
Rehmannia & Astragalus membranaceus and atractylodes rhizome & 80.83 & 97.27 \\
Cornus officinalis Sieb. et Zucc. & Astragalus membranaceus and atractylodes rhizome & 96.04 \\
Moutan bark & Astragalus membranaceus and Paeoniae Radix Alba & & \\
\hline
\end{tabular}

study was 84 months; thus, DPN may have been diagnosed earlier when the patient exhibited clinical manifestations than in patients with a DOD of 10 years. Other studies may have included asymptomatic patients. The average HCY levels in this study were higher than the normal range. Bruce and Young [21] and El Boghdady and Badr [22] found that a high HCY is an important risk factor for DPN. High HCY levels can affect the functions of insulin sensitivity, oxidative stress, nitric oxide, and other pathways of blood vessel and blood vessels and nerves damage $[23,24]$. Therefore, HCY may be an important reference value for the early diagnosis of DPN. However, some scholars believe that HCY is not related to DPN [25]. We found that symptoms due to DPN had a frequency of $75 \%$, which was higher than the findings reported in previous studies, which ranged from $16 \%$ to $65 \%$ $[26,27]$. This difference may be because the patients were hospitalized, indicating that their conditions were more serious.

This study included 216 patients with DPN with qi-yin deficiency syndrome. The results showed the top $5 \mathrm{CHM}$ that were prescribed frequently: Astragalus membranaceus, Ligusticum wallichii, Poria cocos, Radix Rehmanniae, and atractylodes rhizome. Among these herbals, 3 were warm, 1 was cold, and 1 was neutral. Based on these 5 CHM, or 64 


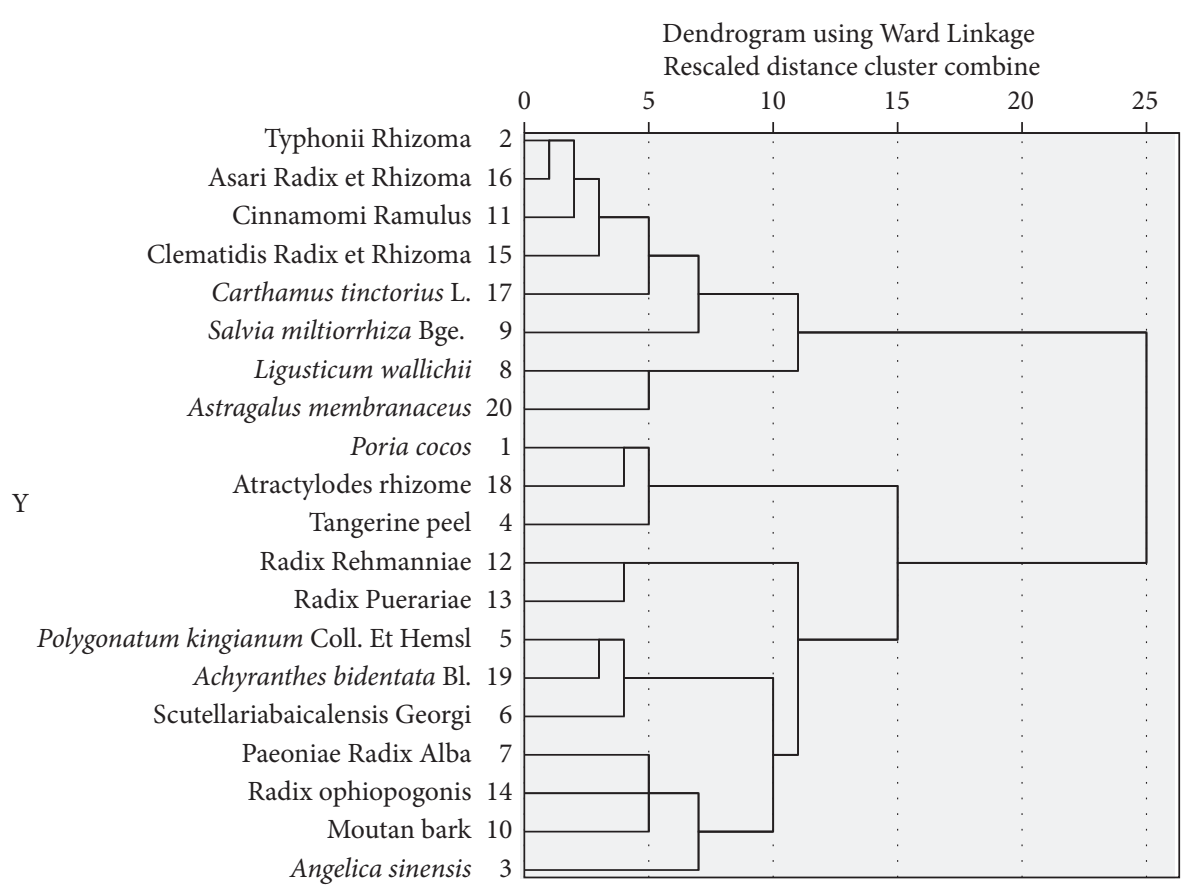

Figure 4: Cluster analysis from CHM.

TABLE 5: CHM and correlation indicators.

\begin{tabular}{|c|c|c|c|}
\hline Drug & Indicators & Support (\%) & Confidence (\%) \\
\hline Astragalus membranaceus and Cinnamomi Ramulus & MDNS $\downarrow$ & 56.74 & 77.35 \\
\hline Ligusticum wallichii and Moutan bark & MDNS $\downarrow$ & 50.25 & 74.31 \\
\hline Radix Rehmanniae, Radix Ophiopogonis, and Radix Puerariae & FPG and $\mathrm{HbA} 1 \mathrm{c} \downarrow$ & 32.43 & 55.21 \\
\hline Astragalus membranaceus and Poria cocos & FPG and $\mathrm{HbAlc} \downarrow$ & 23.15 & 49.36 \\
\hline Salvia miltiorrhiza Bge. and Pinelliae Rhizoma & LDL-C $\downarrow$ & 20.35 & 62.73 \\
\hline
\end{tabular}

warm CHM out of 216 prescriptions, it is believed that the treatment of DPN should be focused on warm CHM. Astragalus membranaceus is sweet and warm. Moreover, it nourishes qi and promotes blood circulation. Furthermore, it is widely used in the treatment of DPN, and it is a monarch CHM. Astragaloside IV and polysaccharides in Astragalus membranaceus decrease glycaemic and regulate blood lipids [28]. Moreover, the antioxidant effect of Astragalus membranaceus improves the symptoms caused by nerve damage [29]. In addition, the dose of Astragalus membranaceus is relatively large, which is consistent with the findings of Feng et al. [30]. Radix Rehmanniae is sweet and cold. It nourishes yin, promotes blood circulation, and moisturizes the skin. Its administration may offer an opportunity to correct epithelial progenitor cell EPC deficits in diabetic patients with damaged blood vessels and nerves [31]. When used clinically, the dose should not be too high to avoid producing a cold or upset stomach. Radix Rehmanniae is a minister and assistant CHM.

These 9 CHM comprise the core of the 216 prescriptions. Astragalus membranaceus is the most core herbal. Cinnamomi Ramulus warms the meridians to stimulate blood to reach the limbs, and it is a minister and assistant CHM. Cinnamaldehyde is the main component of Cinnamomi Ramulus and decreases glycaemic, improves microcirculation, and mediates antiplatelet aggregation and analgesia effects [32]. Astragalus membranaceus and Cinnamomi Ramulus have significant effects on pain in the limbs. This theory may be derived from the application described in the Han Dynasty [33].

The 8 pairs of CHM were identified through correlation analysis. Codonopsis Radix replenishes qi and promotes blood circulation, so that patients do not feel pain or numbness. Radix Ophiopogonis acts by cultivating yin. Analysis of the pharmacological effects of Codonopsis Radix and Radix Ophiopogonis indicates that they decrease glycaemic and are antioxidative $[34,35]$. These effects may relieve clinical DPN symptoms. Astragalus membranaceus and atractylodes rhizome are essential medicines for Yupingfeng powder. Astragalus membranaceus has the effect of two-way regulation of excessive sweat and reduced sweat. These two herbals may also have good clinical effect on antioxidation through polysaccharides in Yupingfeng power [36]. This may regulate abnormal skin sweating in DPN.

Six sets of CHM were identified from the cluster analysis. Poria cocos and atractylodes rhizome are a typical companion CHM pair that improve microcirculatory effects, desensitize channel blocking action to nicotinic acetylcholine receptors, and have antineuronal differentiation actions $[37,38]$. These two CHM also prevent emotional depression 
or hyperactivity caused by DPN symptoms. If patients are in obvious pain, Asari Radix et Rhizoma and Typhonii Rhizoma can be added to relieve pain because these herbals exert sedative, analgesic, and anti-inflammatory effects [39].

There are a series of associations between CHM and indicators. Astragalus membranaceus and Cinnamomi Ramulus or Ligusticum wallichii and Moutan bark are highly related to a decreased MDNS. Feldman et al. [40] reported that the sensitivity and specificity of the clinical portion of the MDNS are $80 \%$ and $95 \%$, respectively. The AUC for MDNS scores was 0.906 [41], the MDNS is a good screening tool for diabetic neuropathy, Astragalus membranaceus, Cinnamomi Ramulus, and Ligusticum wallichii are included in the Huangqi Guizhi Wuwu decoction. Pang et al. [42] and Liang et al. [43] performed a systematic review and meta-analysis reporting that this decoction improves DPN symptoms and ameliorates nerve conduction velocities. However, their studies did not explicitly mention the MDNS. Jin et al. studied the improvement in the MDNS caused by TCM [44]. Radix Puerariae or Poria cocos is highly related to decreased FPG and HbAlc levels when combined with Radix Rehmanniae and Radix Ophiopogonis or Astragalus membranaceus. Lin et al. [35] reported that alcoholic and acetic acid fermentation improve the hypoglycaemic activity of Radix Ophiopogonis. Radix Puerariae has been reported to have antidiabetic effects [45], and these crude herbals reduce the magnitude of increases in glycaemic [46]. Pharmacological studies have indicated that Astragalus membranaceus possesses antioxidant and antidiabetic effects [47]. Poria cocos, as a prebiotic, can potentially prevent or cure metabolic diseases by regulating lipids and glycaemic [48]. If combined with other herbs, Poria cocos makes the hypoglycaemic effect more obvious, which can further regulate HbAlc. Naqvi et al. [49] reported that LDL-C is a related risk factor for DPN. Based on the control of cardiovascular disease risk factors in the context of diabetes and its complications, reduction of LDL-C is recommended as an important goal. In this study, Astragalus membranaceus was associated with Poria cocos when LDL-C was decreased.

This study preliminarily assessed the basic characteristics of DPN patients with qi-yin deficiency syndrome and summarized the characteristics of TCM treatment through data mining technology. In the future, the sample size will be increased for indepth verification of the findings to improve clinical evidence and to better guide clinical practice. The mechanism of action of these CHM prescriptions can also be further explored [50].

\section{Data Availability}

The data used to support the findings of this study are included within the article.

\section{Conflicts of Interest}

The authors declare that they have no conflicts of interest.

\section{Authors' Contributions}

Jindong Zhao, Yan Li, and Zhaohui Fang designed the study and wrote the manuscript. Ling Xin, Min Sun, and Taotao
Bao helped complete the data analysis and improve the English language. Chanjuan Yu, Guobin Shi, Jian Liu, Yingqun $\mathrm{Ni}$, Ruimin $\mathrm{Lu}$, and Yuanyuan $\mathrm{Wu}$ performed the experiment. Jindong Zhao and Yan Li contributed equally to this work.

\section{Acknowledgments}

This study was supported by the National Natural Science Foundation of China (81603574 and 81774286), the Province Science Foundation of Anhui (1708085QH213), the University Scientific Research Projects of Anhui (KJ2019A0442), and Traditional Chinese Medicine Modernization Research (2018YFC1704200).

\section{References}

[1] A. J. Boulton, "Guidelines for diagnosis and outpatient management of diabetic peripheral neuropathy. European Association for the Study of Diabetes, Neurodiab," Diabetes \& Metabolism, vol. 24, no. 3, pp. 55-65, 1998.

[2] A. J. M. Boulton, A. I. Vinik, J. C. Arezzo et al., "Diabetic neuropathies: a statement by the American diabetes association,” Diabetes Care, vol. 28, no. 4, pp. 956-962, 2005.

[3] Q. Yang, Y. X. Zhang, Q. L. Zeng et al., "Correlation between diabetic peripheral neuropathy and sarcopenia in patients with type 2 diabetes mellitus and diabetic foot disease: a crosssectional study," Diabetes, Metabolic Syndrome and Obesity: Targets and Therapy, vol. 13, no. 4, pp. 377-386, 2020.

[4] P. J. Dyck, C. J. Overland, P. A. Low et al., "Signs and symptoms versus nerve conduction studies to diagnose diabetic sensorimotor polyneuropathy: $\mathrm{Cl}$ vs. NPhys trial," Muscle \& Nerve, vol. 42, no. 2, pp. 157-164, 2010.

[5] M. Smieja, D. L. Hunt, D. L. Hunt et al., "Clinical examination for the detection of protective sensation in the feet of diabetic patients," Journal of General Internal Medicine, vol. 14, no. 7, pp. 418-424, 1999.

[6] J.-W. G. Meijer, A. J. Smit, J. D. Lefrandt, J. H. van der Hoeven, K. Hoogenberg, and T. P. Links, "Back to basics in diagnosing diabetic polyneuropathy with the tuning fork!" Diabetes Care, vol. 28, no. 9, pp. 2201-2205, 2005.

[7] M. Bennett, "The LANSS Pain Scale: the Leeds assessment of neuropathic symptoms and signs," Pain, vol. 92, no. 1, pp. 147-157, 2001.

[8] V. Bril and R. A. Buchanan, "Long-term effects of ranirestat (AS-3201) on peripheral nerve function in patients with diabetic sensorimotor polyneuropathy," Diabetes Care, vol. 29, no. 1, pp. 68-72, 2006.

[9] F. Gholami, H. Nazari, and M. Alimi, "Cycle Training improves vascular function and neuropathic symptoms in patients with type 2 diabetes and peripheral neuropathy: a randomized controlled trial," Experimental Gerontology, vol. 131, no. 1, pp. 110799-110805, 2020.

[10] X. W. Yang, W. J. Yao, H. L. Liu et al., "Tangluoning, a traditional Chinese medicine, attenuates in vivo and in vitro diabetic peripheral neuropathy through modulation of PERK/ Nrf 2 pathway," Scientific Reports, vol. 7, no. 1, pp. 1014-1025, 2017.

[11] Y. Wang, Z. Chen, R. Ye, Y. He, Y. Li, and X. Qiu, "Protective effect of Jiaweibugan decoction against diabetic peripheral neuropathy," Neural Regeneration Research, vol. 8, no. 12, pp. 1113-1121, 2013. 
[12] G. J. Fan, H. Y. Huang, Y. P. Lin et al., "Herbal medicine foot bath for the treatment of diabetic peripheral neuropathy: protocol for a randomized, double-blind and controlled trial," Trials, vol. 19, no. 1, pp. 483-491, 2018.

[13] J. Liu, B. Sun, B. Ban et al., "Treatment of type 2 diabetic peripheral neuropathy patients of qi-yin deficiency complicated phlegm-dampness blocking collaterals syndrome by internal application of qigui mixture and external application of qigui huoxue lotion: a clinical study," Chinese Journal of Integrated Traditional and Western Medicine, vol. 34, no. 9, pp. 1053-1058, 2014.

[14] X. Y. Zheng, Guiding Principles for Clinical Research of New Traditional Chinese Medicines, pp. 233-237, China Medical Science and Technology Press, Beijing, China, 2002.

[15] Branch of Chinese Medicine Association, "Guidelines for TCM clinical diagnosis and treatment of diabetic peripheral neuropathy (2016 edition)," Journal of Traditional Chinese Medicine, vol. 58, no. 7, pp. 625-630, 2017.

[16] X. Z. Zhou, S. B. Chen, B. Y. Liu et al., "Development of traditional Chinese medicine clinical data ware house for medical knowledge discovery and decision support," Artificial Intelligence in Medicine, vol. 48, no. 2-3, pp. 139-152, 2010.

[17] National Pharmacopoeia Commission, Pharmacopoeia of the People's Republic of China, China Medical Science and Technology Press, Beijing, China, 2015.

[18] J. Li and Y. Niu, "A research of approximate entropy's clustering analysis in the detection of abnormal flow," Advances in Intelligent and Soft Computing, Springer, Berlin, Germany, pp. 599-605, 2011.

[19] R. Pop-Busui, A. J. M. Boulton, E. L. Feldman et al., "Diabetic neuropathy: a position statement by the American Diabetes Association," Diabetes Care, vol. 40, no. 1, pp. 136-154, 2017.

[20] L. Ang, M. Jaiswal, C. Martin et al., "Glucose control and diabetic neuropathy: lessons from recent large clinical trials," Current Diabetes Reports, vol. 14, no. 9, p. 528, 2014.

[21] S. G. Bruce and T. K. Young, "Prevalence and risk factors for neuropathy in a Canadian First Nation community," Diabetes Care, vol. 31, no. 9, pp. 1837-1841, 2008.

[22] N. A. El Boghdady and G. A. Badr, "Evaluation of oxidative stress markers and vascular risk factors in patients with diabetic peripheral neuropathy," Cell Biochemistry and Function, vol. 30, no. 4, pp. 328-334, 2012.

[23] J. T. Real, J. Folgado, M. Molina Mendez, S. Martinez-Hervás, M. Peiro, and J. F. Ascaso, "Homocisteína plasmática, Lp(a) y marcadores de estrés oxidativo en la vasculopatía periférica del paciente con diabetes tipo 2," Clínica e Investigación en Arteriosclerosis, vol. 28, no. 4, pp. 188-194, 2016.

[24] V. A. Fonseca, L. M. Fink, and P. A. Kern, "Insulin sensitivity and plasma homocysteine concentrations in non-diabetic obese and normal weight subjects," Atherosclerosis, vol. 167, no. 1, pp. 105-109, 2003.

[25] E. K. Hoogeveen, P. J. Konstense, G. D. Valk et al., "Hyperhomocysteinemia is not related to risk of distal somatic polyneuropathy: the Hoorn Study," Journal of Internal Medicine, vol. 246, no. 5, pp. 256-266, 1999.

[26] C. A. Abbott, R. A. Malik, E. R. E. van Ross, J. Kulkarni, and A. J. M. Boulton, "Prevalence and characteristics of painful diabetic neuropathy in a large community-based diabetic population in the U.K," Diabetes Care, vol. 34, no. 10, pp. 2220-2224, 2011.

[27] C. Daousi, S. J. Benbow, A. Woodward, and I. A. MacFarlane, "The natural history of chronic painful peripheral neuropathy in a community diabetes population," Diabetic Medicine, vol. 23, no. 9, pp. 1021-1024, 2006.
[28] L. Z. You, Z. H. Fang, G. M. Shen et al., "Astragaloside IV prevents high glucose-induced cell apoptosis and inflammatory reactions through inhibition of the JNK pathway in human umbilical vein endothelial cells," Molecular Medicine Reports, vol. 19, no. 3, pp. 1603-1612, 2019.

[29] J. Yu, Y. Zhang, S. Sun et al., "Inhibitory effects of astragaloside IV on diabetic peripheral neuropathy in rats," Canadian Journal of Physiology and Pharmacology, vol. 84, no. 6, pp. 579-587, 2006.

[30] L. Feng, W.-K. Liu, L. Deng, J.-X. Tian, and X.-L. Tong, "Clinical efficacy of aconitum-containing traditional Chinese medicine for diabetic peripheral neuropathic pain," The American Journal of Chinese Medicine, vol. 42, no. 1, pp. 109-117, 2014.

[31] J. C. W. Tam, C. H. Ko, K. M. Lau et al., "Enumeration and functional investigation of endothelial progenitor cells in neovascularization of diabetic foot ulcer rats with a Chinese 2herb formula," Journal of Diabetes, vol. 7, no. 5, pp. 718-728, 2015.

[32] P. Subash Babu, S. Prabuseenivasan, and S. Ignacimuthu, "Cinnamaldehyde-a potential antidiabetic agent," Phytomedicine, vol. 14, no. 1, pp. 15-22, 2007.

[33] Y. J. Zheng, F. Yang, L. Han et al., "Efficacy of Chinese herbal medicine in the treatment of moderate-severe painful diabetic peripheral neuropathy: a retrospective study," Journal of Diabetes Research, vol. 2019, Article ID 4035861, 5870 pages, 2019.

[34] J. Y.-W. Chan, F.-C. Lam, P.-C. Leung, C.-T. Che, and K.-P. Fung, "Antihyperglycemic and antioxidative effects of a herbal formulation ofRadix Astragali,Radix CodonopsisandCortex Lyciiin a mouse model of type 2 diabetes mellitus," Phytotherapy Research, vol. 23, no. 5, pp. 658-665, 2009.

[35] W.-L. Lin, W.-W. Su, X.-Y. Cai, L.-K. Luo, P.-B. Li, and Y.-G. Wang, "Fermentation effects of oligosaccharides of Radix Ophiopogonis on alloxan-induced diabetes in mice," International Journal of Biological Macromolecules, vol. 49, no. 2, pp. 194-200, 2011.

[36] D. Wang, B. B. Zhang, X. X. Qu et al., "Microwave-assisted extraction of polysaccharides from Yupingfeng powder and their antioxidant activity," Pharmacognosy Magazine, vol. 11, no. 43 , pp. 546-554, 2015.

[37] T. Y. C. Poon, K. L. Ong, and B. M. Y. Cheung, "Review of the effects of the traditional Chinese medicine Rehmannia Six Formula on diabetes mellitus and its complications," Journal of Diabetes, vol. 3, no. 3, pp. 184-200, 2011.

[38] I. Kimura, "Medical benefits of using natural compounds and their derivatives having multiple pharmacological actions," Yakugaku Zasshi, vol. 126, no. 3, pp. 133-143, 2006.

[39] S. Han, J. Huang, J. Hou, and S. Wang, "Screening epidermal growth factor receptor antagonists from Radix et Rhizoma Asariby two-dimensional liquid chromatography," Journal of Separation Science, vol. 37, no. 13, pp. 1525-1532, 2014.

[40] E. L. Feldman, M. J. Stevens, P. K. Thomas et al., "A practical two-step quantitative clinical and electrophysiological assessment for the diagnosis and staging of diabetic neuropathy," Diabetes Care, vol. 144, no. 8, pp. 1941-1953, 2018.

[41] M. Barbosa, A. Saavedra, M. Severo, C. Maier, and D. Carvalho, "Validation and reliability of the Portuguese version of the Michigan neuropathy screening instrument," Pain Practice, vol. 17, no. 4, pp. 514-521, 2017.

[42] B. Pang, T. Y. Zhao, L. H. Zhao et al., "Huangqi Guizhi Wuwu Decoction for treating diabetic peripheral neuropathy: a meta-analysis of 16 randomized controlled trials," Neural Regeneration Research, vol. 11, no. 8, pp. 1347-1358, 2016. 
[43] L. Liang, X. Wei, M. S. Feng et al., "Huangqi Guizhi Wuwu Decoction for treating cervical radiculopathy: a systematic review and meta-analysis of randomized controlled trials," Medicine (Baltimore), vol. 99, no. 7, pp. 19137-19153, 2020.

[44] D. Jin, W. J. Huang, X. Meng et al., "Chinese herbal medicine TangBi Formula treatment of patients with type 2 diabetic distal symmetric polyneuropathy disease: study protocol for a randomized controlled trial," Trials, vol. 18, no. 1, pp. 631-640, 2017.

[45] H. Jung, A. Kang, S. Kang, Y.-K. Park, and M. Song, “The root extract of Pueraria lobata and its main compound, puerarin, prevent obesity by increasing the energy metabolism in skeletal muscle," Nutrients, vol. 9, no. 1, pp. 33-45, 2017.

[46] T. Yokozawa, H. Y. Kim, and N. Yamabe, "Amelioration of diabetic nephropathy by dried rehmanniae radix (di huang) extract," The American Journal of Chinese Medicine, vol. 32, no. 6, pp. 829-839, 2004.

[47] L. Cheng, G. Zhang, Y. Zhou et al., "Systematic review and meta-analysis of 16 randomized clinical trials of Radix astragali and its prescriptions for diabetic retinopathy," $E v$ idence-Based Complementary and Alternative Medicine, vol. 2013, Article ID 762783, 13 pages, 2013.

[48] S.-S. Sun, K. Wang, K. Ma, L. Bao, and H.-W. Liu, “An insoluble polysaccharide from the sclerotium of Poria cocos improves hyperglycemia, hyperlipidemia and hepatic steatosis in ob/ob mice via modulation of gut microbiota," Chinese Journal of Natural Medicines, vol. 17, no. 1, pp. 3-14, 2019.

[49] S. S. Z. H. Naqvi, S. Imani, H. Hosseinifard et al., "Associations of serum low-density lipoprotein and systolic blood pressure levels with type 2 diabetic patients with and without peripheral neuropathy: systemic review, meta-analysis and meta-regression analysis of observational studies," BMC Endocrine Disorders, vol. 19, no. 1, pp. 125-140, 2019.

[50] J. D. Zhao, Y. Li, M. Sun et al., "The Chinese herbal formula shenzhu tiaopi granule results in metabolic improvement in type 2 diabetic rats by modulating the gut microbiota," Evidence-Based Complementary and Alternative Medicine, vol. 2019, Article ID 6976394, 14 pages, 2019. 